Original Article

\title{
Effects of neurofeedback training on the brain wave of adults with forward head posture
}

\author{
Hyun-Ju OH, PhD, $\mathrm{PT}^{1)}$, Gui-Bin Song, PhD, $\mathrm{PT}^{2)^{*}}$ \\ 1) Department of Physical Therapy, Taegu Science University, Republic of Korea \\ 2) Department of Physical Therapy Yeungnam University College: 170 Hyunchung-ro, Namgu, Daegu \\ 705-703, Republic of Korea
}

\begin{abstract}
Purpose] The purpose of the present study was to examine the effects of neurofeedback training on electroencephalogram changes in the cervical spine in adults with forward head posture through x-ray. [Subjects and Methods] The subjects of the study were 40 college students with forward head posture, randomly divided into a neurofeedback training group (NFTG, $n=20)$ and a control group $(C G, n=20)$. The neurofeedback training group performed six sessions of pottery and archery games, each for two minutes, three times per week for four weeks, while using the neurofeedback system. [Results] There were significant effects within and between groups in terms of the Delta wave, the Theta wave, the Alpha wave, the Beta wave, or the sensory motor rhythm. Especially, the Delta wave, Beta wave, and the sensory motor rhythm were showed significant effects between the groups. [Conclusion] It is thought that neurofeedback training, a training approach to self-regulate brain waves, enhances concentration and relaxation without stress, as well as an increase in attention, memory, and verbal cognitive performance. Therefore an effective intervention method to improve neck pain and daily activities.

Key words: Neurofeedback, Forward head posture, Brain wave
\end{abstract}

(This article was submitted May 14, 2016, and was accepted Jul. 7, 2016)

\section{INTRODUCTION}

Owing to the prevalence and popularity of computers, students and workers are increasingly experiencing musculoskeletal abnormalities in their neck and shoulders ${ }^{1)}$. Using computers and smart phones for many hours, coupled with lack of exercise, may cause stiffness of the muscles in the neck and shoulders, inducing weakness in the soft tissues. Such postural and lifestyle habits lead to forward head posture (FHP), which can cause relative compensation such as increased lordosis in the junction of the skull and neck, and abnormal, consistent muscular contraction inducing changes in the craniocervical junction ${ }^{2}$. Attention is a basic cognitional function necessary for learning and information processing, and it serves as a basic ability essential for educational activity, information acquisition, and social adjustment ${ }^{3}$. Attention and memory are the most basic elements of human cognition; issues with either may affect the seamless acquisition of new information and consistent performance in learning and tasks ${ }^{4}$. Posture affects people in terms of psychological, physical, structural, and functional changes. Specifically, bad posture is thought to increase the possibility of a decline in learning efficiency, attention, and memory. The collum and the brain, in particular, are closely situated in terms of anatomical structures. According to previous research, FHP may induce a reduction in proprioceptive sensibility, in addition to interference between the nerves and the muscles. These problems may, in turn, affect an individual's mind and emotions ${ }^{5}$. Neuro-feedback (NFB) is a type of brain training — based on biofeedback - in which brainwaves are induced and feedback of the induction is provided so that users can control their own brainwaves. It has been researched in various fields, including brain activation training for the elderly and training for sports requiring concentration, such as golf, archery, and shooting ${ }^{6}$. Learning processes, thought, and activity are controlled by cerebral functions, which depend on the activities of many cranial nerves. These activities can be

\footnotetext{
*Corresponding author. Gui-Bin Song (E-mail: guibinlove@hanmail.net)

(C)2016 The Society of Physical Therapy Science. Published by IPEC Inc.

This is an open-access article distributed under the terms of the Creative Commons Attribution Non-Commercial No Derivatives (by-nc-nd) License $<$ http://creativecommons.org/licenses/by-nc-nd/4.0/>.
} 
identified with the help of electroencephalogram (EEG). When compared to other neuroimaging techniques for research, the EEG is a short-term, noninvasive method that directly shows changes in cerebral function. From a cost perspective, it has the advantage of being able to provide various useful data based on a short-term test.

In this study, a neuro-feedback training program to a study sample with unstable postures and identified the influence of neuro-feedback training program on the subjects by means of an EEG, on the assumption that the FHP was due to malalignment of the cervical joints and was possibly affecting the subjects' attention.

\section{SUBJECTS AND METHODS}

The subjects of this study were students from a university located in Gyeongsangbuk-do, South Korea, whose cervical lordosis angle was $21^{\circ}$ or less ${ }^{7}$, whose anterior weight bearing (AWB) of the head was $15 \mathrm{~mm}$ or more ${ }^{8}$, and whose cervical extension range of motion (ROM) was $70^{\circ}$ or less. They understood the purpose and method of the study, and voluntarily provided consent before the experiment. Those who were periodically undergoing treatment or taking drugs for pain reduction, and those with neurological problems were excluded from the experiment. Table 1 shows the general characteristics of the subjects. All patients understood the purpose of this study and provided written informed consent prior to participation in accordance with the ethical standards of the Declaration of Helsinki.

The subjects were randomly divided into a neuro-feedback group and a control group, and underwent a radiographic check and a nondestructive inspection (NDI). After a four-week application of intervention three times per week for each group, the subjects underwent another radiographic check and NDI. Neuro-feedback training was applied to the neuro-feedback group using the Neuro-Feedback System (Braintech Corp., Korea) developed by the Korean Research Institute of Jungshin Science. The training was performed in a place where light and noise were blocked. After taking a rest for ten minutes, each subject wore a headband in such a way that the FPz, as the center electrode, adhered to the middle of the forehead above the prefrontal region, and the reference electrode adhered to the left earlobe. As a training exercise for improving attention, the subjects performed a cup-making game and a mind arrow game. In the cup-making game, the subject had to collect glass pieces to make a cup; the more cups made, the higher the score. When the brainwaves were stable enough to satisfy the training conditions, a signal sound indicated that a cup was complete. If the brainwaves were unstable, the program stopped. The game was performed six times for two minutes each time. The mind arrow was a game of archery using brainwaves. When the brainwaves of the subject satisfied the training conditions and a signal sound was made, the subject could shoot an arrow with the aim of hitting the target in the center. Fifteen arrows could be shot for two minutes, and the total score indicated the results of the brainwave training after six sessions, each lasting twelve minutes.

Radiographic inspection was conducted to identify the following: the absolute rotation angle (ARA) ${ }^{9)}$ for measuring lordosis of the neck; anterior weight bearing $(\mathrm{AWB})^{10)}$ to investigate forward head posture; and range of flexion and extension motions(PFEM $)^{11)}$ to identify the angle of flexion and extension. In general, it is the brainwaves of the frontal lobe that are used for recording purposes. In this study, we measured the Delta $(\delta)$ wave, the Theta $(\theta)$ wave, the Beta $(\beta)$ wave, and the sensory motor rhythm (SMR) for recording purposes. We followed the international 0-20 system to locate scalp electrodes for the brainwave recording. When the total ratio is determined as 50, the ratio of the electrodes is 20:20:10 between the parietal region and the nasion in a forward direction, between the parietal region and the inion in a rearward direction, and between the parietal region and the upper point of both auricles laterally ${ }^{12}$.

SPSS 14.0 for Windows was used to statistically process the data in this study. An independent t-test was performed for pretest-posttest within-group comparison and for pretest-posttest difference comparison between the groups. For level of statistical significance, $\mathrm{p}=0.05$.

\section{RESULTS}

As seen in Table 2, the neuro-feedback group showed significant differences in the $\delta$ wave, the $\theta$ wave, the $\alpha$ wave, the $\beta$ wave, and the SMR $(p<0.05)$ in the pretest-posttest within-group comparison, while the control group showed no significant difference in the $\delta$ wave, the $\theta$ wave, the $\alpha$ wave, the $\beta$ wave, or the SMR in the comparison ( $>0.05$ ). As for the pretestposttest difference comparison between groups, significant differences were found in the $\delta$ wave, the $\beta$ wave, and the SMR $(\mathrm{p}<0.05)$ (Table 2).

\section{DISCUSSION}

Jang ${ }^{13)}$ reported that neuro-feedback training using brainwaves may generate positive brainwaves due to the force of one's will to feedback one's own physical reactions with a view to modifying behavior. Brainwave examination by EGG is a noninvasive method that displays functional changes in the cerebrum and provides various useful data within a short time $^{14)}$. A $\beta$ wave faster than $13 \mathrm{~Hz}$ is activated when a normal person is excited or concentrates on a certain task, and this is usually displayed in the frontal and parietal regions. The SMR becomes predominant when a person pays attention to a relatively simple task, while the activities of the sensory motor cortex are minimized without physical movement. The $\alpha$ 
Table 1. General characteristics of each group (Mean \pm SD)

\begin{tabular}{lcc}
\hline & NFTG $(\mathrm{n}=20)$ & CG $(\mathrm{n}=19)$ \\
\hline Male/Female & $9 / 11$ & $9 / 10$ \\
Age (years) & $20.9 \pm 3.3$ & $22.8 \pm 4.4$ \\
Height $(\mathrm{cm})$ & $168.5 \pm 9.2$ & $167.6 \pm 8.7$ \\
Body weight $(\mathrm{kg})$ & $59.3 \pm 11.5$ & $59.5 \pm 11.4$ \\
\hline
\end{tabular}

NFTG: neurofeedback training group; CG: control group

Table 2. Comparison of the change of brain wave between the neurofeedback training group and the control group (mean $\pm \mathrm{SD}$ )

\begin{tabular}{lrrrr}
\hline & \multicolumn{2}{c}{ NFTG } & CG \\
\hline DEF & Pre-test & Post-test & Pre-test & Post-test \\
TEF & $17.3 \pm 0.5$ & $14.9 \pm 3.6^{* a}$ & $17.2 \pm 0.7$ & $17.1 \pm 3.7$ \\
ALF & $29.5 \pm 0.2$ & $27.8 \pm 3.5^{*}$ & $29.4 \pm 0.7$ & $28.6 \pm 3.7$ \\
BEF & $33.0 \pm 0.4$ & $36.0 \pm 7.3^{*}$ & $33.2 \pm 0.7$ & $35.7 \pm 6.8$ \\
SMRF & $20.2 \pm 0.4$ & $27.3 \pm 8.2^{* a}$ & $20.2 \pm 0.4$ & $21.3 \pm 2.3$ \\
\hline
\end{tabular}

*Significant difference from the pre-test at $<0.05$. ${ }^{a}$ Significant difference in gains between the two groups. $\mathrm{p}<0.05$. NFTG: neurofeedback training group; CG: control group; DEF: delta frontal; TEF: theta frontal; ALF: alpha frontal; BEF: beta frontal; SMRF: sensory motor rhythm frontal

wave is a basic brainwave reflecting the stability of the brain, and it can be observed when the balance of brain activities is focused or attention is needed. Conversely, the $\beta$ wave is prominent when a person is experiencing worry or anxiousness. The $\delta$ wave is induced, not in a conscious state but in an unconscious state, and it is associated with intuition. As seen in a patient with a head injury, the $\delta$ wave in an arousal state slows down data processing in the brain to interrupt conscious activities ${ }^{15)}$. Penistone ${ }^{15)}$ reports that regular relaxation or meditation training may reduce the low frequency potential and increase the activation potential of the $\alpha$ wave to induce a stable mentality.

In this study, the neuro-feedback training group showed significant increases in the $\delta$ wave, the $\theta$ wave, the $\alpha$ wave, the $\beta$ wave, and the SMR after the intervention, while the control group showed no significant increase. As for the pretest-posttest difference comparison between the groups, significant differences were observed in the $\delta$ wave, the $\beta$ wave, and the SMR. After the intervention, the neuro-feedback training group showed significant changes in the $\alpha$ wave and the $\delta$ wave, indicating that their attention was enhanced because the brainwaves were stabilized by the brain training. The results of this study were supported by Penistone ${ }^{15}$ ) in research reporting that regular relaxation or meditation training increases the activation potential of the $\alpha$ wave to induce a stable state of the brain. The $\theta$ wave and the $\beta$ wave also showed significant changes in the neuro-feedback training group, results that were similar to those of Lee ${ }^{16)}$ who reported a reduced $\theta$ wave in the frontal lobe and the parietal lobe after training, and those of Seo and Park ${ }^{17)}$, in which cognitive anxiety declined and attention increased in normal people when training to maximum performance competency. Such changes may be common characteristics of brainwave types inducing increased mental attention. In the performance of a task, the $\theta$ wave and the $\beta$ wave tended to be reduced, indicating an increase in attention and favorable achievement ${ }^{14)}$. Sterman ${ }^{18)}$ reports that the SMR is closely related to attention and indicates that an increase in the SMR may induce changes to a state of attention. The results of this study are similar to those of Sterman. In this context, neuro-feedback training may be helpful in stress relaxation, enhancement of attention, and a more stable, efficient state of brain functions that will optimize brain performance.

In this study, brainwave changes by means of neuro-feedback training in adults with forward head posture (FHP). After the neuro-feedback training, the subjects showed significant increases in the $\delta$ wave, the $\theta$ wave, the $\alpha$ wave, the $\beta$ wave, and the SMR. FHP induces muscular tension and malalignment of the cervical structures, causing stress and a decline in attention due to transformation of the body structure. Our assumption was that after the neuro-feedback training, the subjects with FHP might experience a stable mentality and relaxation without stress, as well as an increase in attention, memory, and verbal cognitive performance. We sought to verify these assumptions based on changes in their brainwaves during the training. Although direct effects on attention were not measured in this study, it might be said that the training positively affected attention. Consistent studies may be needed in the future to identify various methods for measuring brainwaves in many parts of the brains of patients with different disorders. 


\section{REFERENCES}

1) Mekhora K, Liston CB, Nanthavanij S: The effect of ergonomic intervention on discomfort in computer users with tension neck syndrome. Int J Ind Ergon, 2000, 26: 367-379. [CrossRef]

2) Harrison DE, Harrison DD, Betz JJ, et al.: Increasing the cervical lordosis with chiropractic biophysics seated combined extension-compression and transverse load cervical traction with cervical manipulation: nonrandomized clinical control trial. J Manipulative Physiol Ther, 2003, 26: 139-151. [Medline] [CrossRef]

3) Rizzo AA, Buckwalter JG, Humphrey L, et al.: The virtual classroom: a virtual reality environment for the assessment and rehabilitation of attention deficits Cyberpsychol Behav, 2003, 3: 483-500. [CrossRef]

4) Nethertom DS, Holmes D, Walker CE: Child and adolescent psychological disorders. New York: Oxford University Press, 1999 , pp 57-86.

5) Nicholas MK: Mental disorders in people with chronic pain: an international perspective. Pain, 2007, 129: 231-232. [Medline] [CrossRef]

6) Chung CH: Effects of concentration training with brainwave biofeedback on tennis performance proceedings of 10th world congress of sport psychology, 2000, 94-96.

7) Owens E, Hoiris K: Cervical curvature assessment using digitized radiographic analysis. J Chiropr Res, 1990, 4: 47-62.

8) Oakley PA, Harrison DD, Harrison DE, et al.: Evidence-based protocol for structural rehabilitation of the spine and posture: review of clinical biomechanics of posture (CBP) publications. J Can Chiropr Assoc, 2005, 49: 270-296. [Medline]

9) Ro HL, Gong WT, Ma SY: Coreelations between and absolute rotation angle, anterior weight bearing, range of flexion and extension motion in cervical herniated nucleus pulposus. J Phys Ther Sci, 2010, 22: 447-450. [CrossRef]

10) Lee H, Nicholson LL, Adams RD, et al.: Development and psychometric testing of Korean language versions of 4 neck pain and disability questionnaires. Spine, 2006, 31: 1841-1845. [Medline] [CrossRef]

11) Gong WT, Hwang Bo G, Lee YM: The effects of Gong's mobilixation on cervical lordosis, forward head posture, and cervical ROM in abnomal posture of the cervical spine of college students. J Phys Ther Sci, 2011, 23: 531-534. [CrossRef]

12) Lee JA: The effect of Synchronicity music meditation on brain wave and psychological changes. Counseling psychology major. The Graduate School of Changwon National University, 2004.

13) Jang JG: The effects of the concentration training through brain wave biofeedback on the track and field performance. Korea Sport Research, 2005, 16: $151-158$

14) Florence G, Guerit JM, Gueguen B: Electroencephalography (EEG) and somatosensory evoked potentials (SEP) to prevent cerebral ischaemia in the operating room. Neurophysiol Clin, 2004, 34: 17-32. [Medline] [CrossRef]

15) Penistone EG, Kulkosky P: Alcoholic personality and alpha-thera brain wave training. Adv Med Psychother, 1991, 4: 1-14.

16) Lee YH: The effect of attention and memory on alpha wave-relax training program in students with cerebral palsy. Special education, Daegu University, 2000.

17) Seo HS, Pack HB: Clinical applications of neurofeedback treatment for insomnia. Korea Sleep Society, 2007, 14: 79-85.

18) Sterman MB: Sensorimotor EEG operant conditioning: experimental and clinical effects. Pavlov J Biol Sci, 1977, 12: 63-92. [Medline] 